OPEN ACCESS

Edited by: John Maher, King's College London, United Kingdom

Reviewed by: Tessa Gargett,

Royal Adelaide Hospital, Australia Amorette Barber, Longwood University, United States

*Correspondence: Gwo Ho

gwo-yaw.ho@monash.edu

${ }^{t}$ These authors have contributed equally to this work

Specialty section:

This article was submitted to Cancer Immunity and Immunotherapy,

a section of the journal

Frontiers in Immunology

Received: 25 February 2021 Accepted: 22 March 2021

Published: 13 April 2021

Citation:

Wu JWY, Dand S, Doig L, Papenfuss AT, Scott CL, Ho G and

Ooi JD (2021) T-Cell Receptor

Therapy in the Treatment of Ovarian Cancer: A Mini Review.

Front. Immunol. 12:672502. doi: 10.3389/fimmu.2021.672502

\section{T-Cell Receptor Therapy in the Treatment of Ovarian Cancer: A Mini Review}

\author{
Jessica W. Y. Wu ${ }^{1}$, Sudiksha Dand ${ }^{1}$, Lachlan Doig ${ }^{2}$, Anthony T. Papenfuss ${ }^{2}$, \\ Clare L. Scott ${ }^{2}$, Gwo Ho ${ }^{1,3^{*}+}$ and Joshua D. Ooi ${ }^{1+}$ \\ ${ }^{1}$ School of Clinical Sciences, Monash University, Clayton, VIC, Australia, ${ }^{2}$ Walter and Eliza Hall Institute of Medical Research, \\ Parkville, VIC, Australia, ${ }^{3}$ Department of Oncology, Monash Health, Clayton, VIC, Australia
}

Ovarian cancer, in particularly high-grade serous ovarian cancer (HGSOC) and ovarian carcinosarcoma (OCS), are highly aggressive and deadly female cancers with limited treatment options. These tumors are generally unresponsive to immune check-point inhibitor $(\mathrm{ICl})$ therapy and are referred to as immunologically "cold" tumors. Cell-based therapy, in particular, adoptive T-cell therapy, is an alternative immunotherapy option that has shown great potential, especially chimeric antigen receptor T cell (CAR-T) therapy in the treatment of hematologic malignancies. However, the efficacy of CAR-T therapy in solid tumors has been modest. This review explores the potential of another cell-based therapy, T-cell receptor therapy (TCR-T) as an alternate treatment option for immunological "cold" OC and OCS tumors.

Keywords: T-cell receptor, tumor neoantigen, cell-based therapy, ovarian cancer, immunotherapy

\section{INTRODUCTION-OVARIAN CANCER; HIGH GRADE SEROUS OVARIAN CANCER AND OVARIAN CARCINOSARCOMA}

Ovarian cancer $(\mathrm{OC})$ is the $8^{\text {th }}$ most commonly diagnosed cancers among women globally, with epithelial ovarian carcinomas being the most common type of OC diagnosed $(1,2)$. Unfortunately most OC, more than $75 \%$ of cases, are diagnosed at late stages (stage III to IV) due to their indolent presentation and the lack of effective screening tools.

The majority of epithelial ovarian carcinomas are high-grade serous ovarian carcinomas (HGSOC), which are of aggressive nature and associated with poor 5-year overall survival of less than 35 percent (2) (see Figure 1A). HGSOC is a heterogenous group of cancers that can be molecularly subtyped based on their gene expression profile (3). Ovarian carcinosarcomas (OCS) 
A

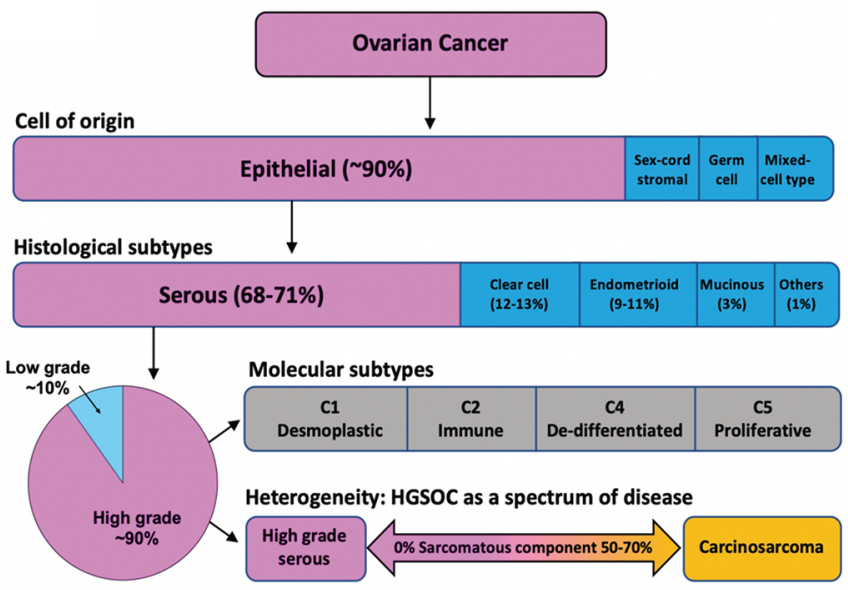

C

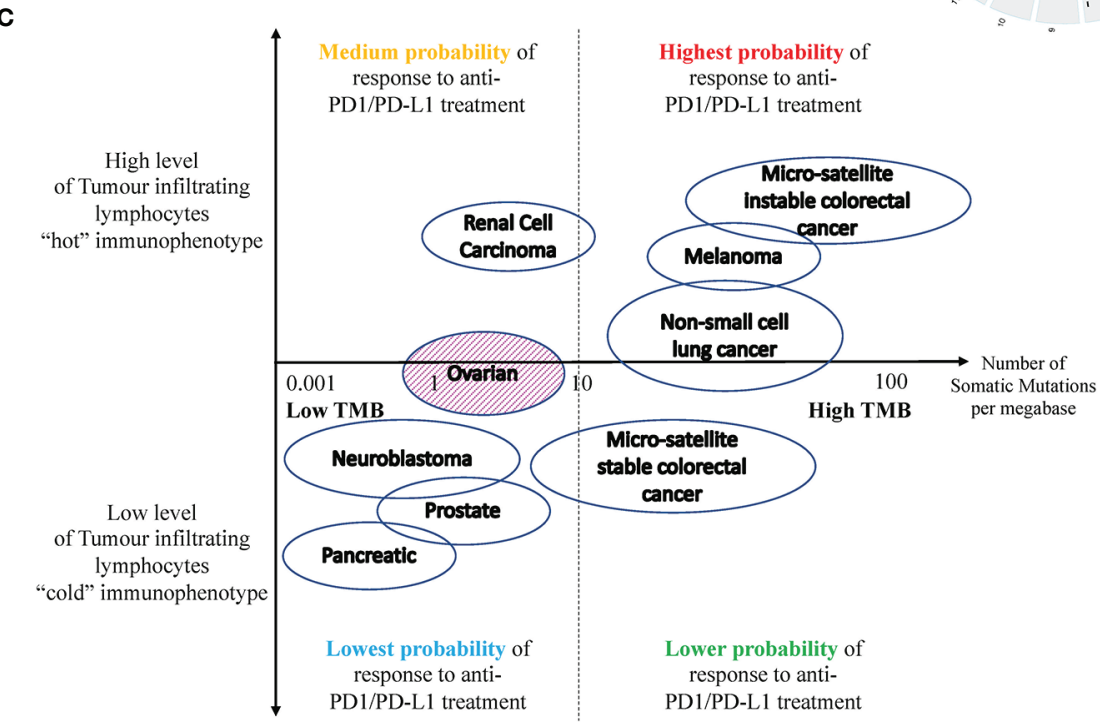

B
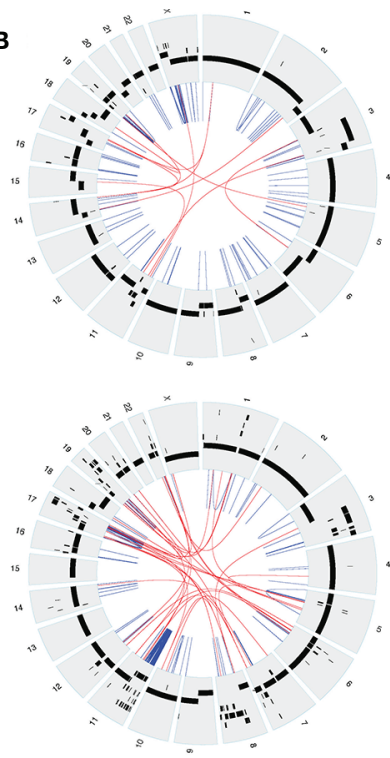

FIGURE 1 | (A) Ovarian cancer can be classified into four subtypes based on their cell of origin and histological characteristics. Epithelial OC is the most common subtype of OC, which can be further divided into 5 histological groups. HGSOC is the most common subtype of EOC accounting for about $70 \%$ of cases and is associated with the poorest prognosis. HGSOC can be further molecularly sub-classified based on its gene expression profile, into 4 groups (C1, C2, C4 and C5). Ovarian or fallopian tube derived carcinosarcoma (O/FTCS) is a rare subtype of OC and may belong within the spectrum of HGSOC tumor. (B) CIRCOS plots of a patient with HGSOC (top) and a patient with OCS (bottom) with low tumor mutation burden - demonstrating a high degree of chromosomal instability (C) Schematic diagram of tumors with high and low tumor mutation burdens versus inflamed and non-inflamed immunophenotypes based on their T-cell and IFN signature. Although some ovarian cancers may have low TMB, these tumors may still respond to ICl if they are associated with a "hot" immunophenotype. Completely "cold" tumors are those associated with low TMB and cold immunophenotype features, such as pancreatic, prostate cancer and some OcS.

are a rare subtype of OC with a worse prognosis (4). It is argued that OCS may be a subtype of HGSOC given that they share common genomic features and cell of origin (5).

Improvements in survival outcomes for OC patients worldwide over the past two decades have been minimal, with the exception of the recent introduction of Poly (ADP-ribose) polymerases inhibitors (PARPi) (6). In recent years, in vivo preclinical testing of novel therapeutics for OC have been aided by an improved understanding of tumor biology - built upon decades of technological advancements in genetic engineering and refinements in mouse models, including the patient-derived xenograft (PDX) model.

Currently, cell-based immuno-oncology for solid tumors such as in OC is an emerging field, which holds a wealth of unrealized potentials. This review will provide an overview of cell-based immunotherapies for OC, in particular T-cell receptor cell-based immunotherapy approach targeting tumor neoantigens (TNA) to treat immunologically "cold" HGSOC and OCS. 
HIGH-GRADE SEROUS OVARIAN CANCER AND OVARIAN CARCINOSARCOMA ARE OFTEN REGARDED AS IMMUNOLOGICALLY "COLD" TUMORS

Immunotherapies (IO), in Particular, Immune Checkpoint Inhibitors (ICI), Are Often Ineffective When Delivered as a Single Agent in OC, Particularly in HGSOC

Immunotherapies are increasingly being explored as a potential treatment option for OCs, given the longevity of responses observed in responsive tumors (7-9). These responsive tumors are often referred to as immunologically "hot" tumors. ICIs are a traditional class of immunotherapies used to target tumors that disrupt the immune checkpoint pathways for immune evasion. ICIs such as anti-PD-1/PDL-1 monoclonal antibodies (e.g., nivolumab, pembrolizumab) targeting the PD-1/PD-L1 axis or anti-CTLA-4 antibodies (e.g., ipilimumab) have shown a great degree of clinical efficacy as single agent or in-combination in non-small cell lung cancer and melanoma respectively with the list of cancer subtypes that are being or under investigation is rapidly expanding (currently including head and neck carcinoma, renal cell carcinoma, uterine carcinoma, colorectal and upper gastro-intestinal carcinoma, bladder carcinoma, hepatocellular carcinoma and small cell lung cancer) $(10,11)$. These therapies are associated with acceptable toxicities reported in multiple Phase II/III clinical trials (12).

However, ICI efficacy is limited in HGSOCs. These tumors are often not mutation-driven and tend to have low rates of tumor mutation burden (TMB) (13-16).. The use of single agent ICIs has resulted in modest response rates in most clinical trials involving HGSOC, however, those who responded, achieved significant clinical benefit. Hence, these findings serve to inform and outline the importance of selecting the right tumor groups within HGSOC for ICI, in particular identifying biomarkers to predict ICI responses. Another approach to improve the response rate is to use ICI with a combinatory synergistic partner such as in combination with a PARPi or a mitogen-activated protein kinase kinase inhibitor (MEKi) $(17,18)$.

Due to OCS rarity, the efficacy of IO, in particularly ICI, is relatively unknown. Given that OCS share similar genomic landscape with HGSOC, it is likely that OCS is also relatively immunologically "cold" (19).

\section{HGSOC and OCS Are Often Regarded as "Class C" Tumors Where Tumorigenesis Is Driven by Chromosomal Instability Rather Than Mutation}

Almost all HGSOCs demonstrate the loss of maintenance of their genome integrity leading to extreme genomic instability (20). This is demonstrated by the remarkable degree of genomic disarray seen in all these tumors characterized by high level of genomic structural variability, associated with frequent somatic copy number alterations (SCNA), in both gains and losses (21-23). Therefore, these tumors are classified as "Class C" malignancy, together with a large fraction of lung (LUSC) and head and neck (HNSC) squamous cell carcinoma and endometrioid tumor of the serous subtype (UCEC-serous) (24). The "Class C" tumors in general have high levels of chromosomal instability as well as enrichment of TP53 mutations, which in turn contribute to the tumor genomic instability. The opposite is the "Class M" tumor, which is predominantly driven by mutation.

The genomic instability is highly relevant for HGSOC tumorigenesis because chromosomal structural change is one of the important mechanisms for tumor suppressor gene (TSG) inactivation either through heterozygous or homozygous loss (25), or gene breakage (26). Similarly, high gene copy number gain can result in differential expression of oncogenes, such as $M Y C N$ and CCNE1, which are relevant in the initiation of tumorigenesis and as mechanisms of drug resistance $(3,26-28)$.

Therefore, HGSOC tumorigenesis driven by loss of TSG and/ or over-expression of oncogenes, which are normal genes with specific function in normal tissue whose expressions are well regulated within non-malignant cells. Hypothetically, the loss of TSG and over-expression of oncogenes should not make these tumors immunogenic. These tumorigenic processes do not result in expression of abnormal mutant proteins or peptides that would be recognizable by the body's immune system.

OCS tumorigenesis is thought to be based on the "conversion hypothesis" of sarcomatous transformation of a differentiated cancer cell-type (carcinoma) into undifferentiated (sarcomatous) malignancy $(29,30)$. It is generally accepted that the transformation is not driven by somatic mutation, but via reprogramming of gene expression and associated with high level of genomic instabilities (see Figure 1B). In keeping with HGSOC, OCS should also not be immunogenic.

\section{Tumor Mutational Burden Is Often Low in HGSOC (and/or OCS) and May Be One of the Reason for the Immunological "Cold" Phenotype}

Tumor mutational burden (TMB) is defined as the total number of somatic mutations per coding area of a tumor genome (31). $\mathrm{TMB}$ has been regarded as an emerging potential clinical biomarker associated with response to immune checkpoint inhibitor (ICI) therapy (32). A higher TMB is commonly observed in cancers associated with mutagens, such as ultraviolet light exposure in melanoma and smoking in nonsmall-cell lung cancer (NSCLC). The prevalence of high TMB was seen in approximately $52 \%$ of patients with melanoma and $38 \%$ to $42 \%$ of patients with NSCLC. High TMB or TMB-H is often defined as detection of more than 10 mutations per megabase of $\mathrm{DNA}$, denoted as mut/Mb. In general sense, tumors with high TMB are likely to express more neoantigens that may be one of the factors driving anti-tumor immunity thus making these tumors "hot" and more responsive to anti-PD-1/ PD-L1 therapies (33). It is important to take into consideration that ICI therapies mode of action is not to initiate T-cells activities but to re-invigorate already tumor-reactive T-cells (33).

In contrast, due to the drivers underlying HGSOC and OCS tumorigenesis, HGSOC and OCS are often associated with lower 
TMB or TMB-L. Tumors with TMB-L have been linked to poorer responses to ICI. In contrast, TMB-H tumors are associated with better responses to ICI as they potentially have higher levels of neoantigens that can be recognized by the immune system $(32,33)$. However, not all TMB-L tumors are immunologically "cold" as the presence of tumor infiltration lymphocytes (TILs) or inflamed T-cells may improve tumor response to ICI - see Figure 1C.

There are a number of other factors that have been proposed to contribute to the "cold" tumor immune-phenotype in HGSOC. These include the expression of endothelin B receptor (34) and Fas ligand (35) within the tumor endothelium, elevated levels of vascular endothelial growth factors (VEGF) which are commonly seen in HGSOC (36), and the presence of epigenetic silencing of Th1-type chemokines (37). The overexpression of endothelin B receptor, Fas ligand and VEGF altered the tumor micro-environment, in particularly affecting the tumor endothelial barrier to hinder T-cell homing and infiltration into the tumor. Similarly, the repression of Th1-type chemokines expression, such as CXCL9 and CYCL10, leads to impaired T-cell trafficking into the tumor. The reduction or absence of TILS compounds the lacks of ICI activity. Cold OC tumors are also shown to be enriched for a class of genomic alterations known as foldback inversions, resulting in high level of chromosomal translocation (38).

However, not all OC or HGSOC are cold immunologically (see Figure 1C). Those HGSOC that are immunologically "hot" are associated with mutations in BRCA1 and, in some studies, $B R C A 2(39,40)$. Studies have shown that within immunological cold tumors, there are tumor sites which showed enhanced signs of immune editing, including neoantigen depletion and allelespecific loss of MHC class I, suggesting that these tumors were immunologically "hot" to begin with (38).

\section{ADOPTIVE T-CELL THERAPIES (ATC); A POTENTIAL APPROACH TO TREAT IMMUNOLOGICALLY "COLD" OVARIAN CANCERS}

\section{Cell-Based Therapy May Be an Alternative Immunotherapy Option for Immunologically "Cold" OCs}

Cell-based therapy strategy includes the adoptive transfer of autologous antigen-specific T-cells, which have undergone ex vivo modification and expansion, with the aim of achieving a targeted immune response (41). This can be achieved with the utilization of ex vivo expanded tumor infiltrating lymphocytes (TIL) with high tumor-specific reactivity, or genetically modified peripheral blood mononuclear cells (PBMC) (41).

Earlier attempts have been relatively unsuccessful due to various challenges posed by these approaches in solid tumors, mainly the small number of invasive TILs within immunologically "cold" tumors and the lack of anti-tumor ability of the body's immune system (41). However, recent advances have shown that the low survival and migration of $\mathrm{T}$ cells can be overcome, and immune evasion, at least in hematological malignancies, can be circumvented by $\mathrm{T}$-cell genetic engineering (42).

With respect to generating ATC with improved OC antigen specificity, peripheral blood lymphocytes can be genetically modified to express: (i) a T cell receptor (TCR) with specificity for a tumor-restricted peptide presented by a given HLA molecule $(43,44)$, or (ii) a "chimeric antigen receptor" (CAR) comprising an antigen-binding domain (typically derived from an antibody) linked via a transmembrane domain to one or more intracellular signaling domains derived from the $\mathrm{T}$ cell receptor complex and associated co-stimulatory molecules (45).

\section{Chimeric Antigen Receptor T Cell Therapy Is a Well-Developed Approach to Treat Hematologic Malignancies}

Chimeric antigen receptors (CARs) redirect $\mathrm{T}$ cells to recognize cell surface antigens in an HLA-independent manner. By doing so, it has the potential of becoming an "off-the-shelf" universal approach to treat wide range of tumors expressing the appropriate cell surface antigens. To achieve this, CAR-T cells utilize antibody fragments that bind to specific antigens on the surface of cancer cells.

CAR-T cell therapy has been well studied and is approved for the treatment of various hematological malignancies (38). These include the treatment of relapsed or refractory B-cell precursor acute lymphocytic leukemia (ALL) in patients $\leq 25$ years old (46) approved by the Food and Drug Agency (FDA) in August 2017 and the treatment of adults with relapsed or refractory large Bcell lymphoma, including diffuse large B-cell lymphoma (DLBCL), after two or more lines of systemic therapy (47).

Despite CAR-T therapy successes in hematologic malignancies, the efficacy in solid tumors is less dramatic and is confounded by the associated risk of cytokine release storm (CRS) and other significant immunologic toxicities $(48,49)$. Unlike for hematological malignancies, it is harder to justify the use of CAR-T in solid tumors due to its much lower response rates and associated high cost. Despite this, there are multiple on-going or completed clinical trials of CAR-T therapies in OC targeting MUC1, MUC16, mesothelin, or folate receptor $\alpha$ (50).

\section{T Cell Receptor Therapy Is an Alternate Cell-Based Therapy With Great Potential for Treatment of Solid Tumors}

$\mathrm{T}$ cell receptors (TCRs) use T-cell antigen receptors, which consist of alpha and beta chains, to recognize polypeptide fragments presented by major histocompatibility complexes (MHC) molecules (51). The generation of TCR-T involves a transfer of TCR genes from an activated T-cell to a naïve T-cell. In doing so, the TCR binding to tumor antigens can be genetically modified to enhance specificity and affinity to desired cancer antigens (51). Unlike CAR-T therapy, whose target antigens are only cell surface proteins, TCR-T cell therapy can recognize intracellular antigen fragments as well as surface proteins as long as these are presented by $\mathrm{MHC}$ 
molecules. However, this also means that TCR-T cell therapy is $\mathrm{MHC}$ restricted and depends on the presentation by $\mathrm{MHC}$ molecules to recognize targets and activate $\mathrm{T}$ cell function. There lies both the advantages and disadvantages of TCR-T over CAR-T therapies (49). Furthermore, the somatic loss of HLA-1 in immunologically "cold" tumor will also have a negative impact on the efficacy of TCR-T (52).

CD8+ cytotoxic $\mathrm{T}$ cells play crucial role in the killing of cancerous or virally infected cells. The CD8+ T cells that are used in TCR-T therapy retain all their natural auxiliary molecules of the TCR signal transduction pathway. Therefore, TCR-T cells can be fully activated even when a very small amount of antigen is present, resulting in effective antigen-specific killing (51).

Furthermore, there are many downstream co-stimulatory factors involved in TCR signaling, including the upregulation of anti-apoptotic factors such as B cell lymphoma 2 (BCL-2), $\mathrm{BCL}$ extra-large $(\mathrm{BCL}-\mathrm{xL})$ and $\mathrm{BCL} 2$-related protein $\mathrm{A} 1$ (BCL2A1). These anti-apoptotic factors promote $\mathrm{T}$ cell activation and more importantly their survival during this process (53). Although, there are also confounding coinhibitory factors within the T-cell system to extinguish $\mathrm{T}$ cell signaling in order to keep their cytotoxic activities in check, such as cytotoxic T-lymphocyte antigen 4 (CTLA-4) and programmed cell death protein 1 (PD-1), these co-inhibitory factors can be circumvented with anti-PDL- 1 and anti-CTLA-4 compounds that are widely available in the clinical settings (49).

Lastly, the bystander effect is largely unappreciated in cell based therapy in solid tumor. It has been observed that significant numbers of $\mathrm{T}$ cells are activated in a $\mathrm{T}$ cell receptorindependent and cytokine-dependent manner, a phenomenon referred to as "bystander activation" during T-cell cytotoxic process. The mechanisms of the bystander effect are unclear, but the innate inflammatory cytokines, such as IL-18 and IL-15, are thought to play crucial roles for inducing bystander activation during infection (54). Bystander activation leads to host injury mediated by exerting a higher level cytotoxicity that is further facilitated by natural killer cell-activating receptors, such as NKG2D, and cytolytic molecules, such as granzyme B. Therefore, hypothetically a small number of activated CD8+ T cells infiltrating into a "cold" tumor may exert both $\mathrm{T}$ cell receptor dependent and independent cytotoxic effects on the cancer cells. There is evidence that the bystander effect has been demonstrated to be less profound in relation to CAR-T in solid tumor seen in syngeneic mouse cancer models that is not augmented by co-administration of anti-PD-1 or anti-CTLA-4 agents (55).

\section{T-CELL RECEPTOR TRANSDUCED T CELL THERAPY IN OVARIAN CANCER: CURRENT THERAPEUTIC LANDSCAPE}

T-cell receptor transduced-T cell (TCR-T) therapy in OC is currently in early phase clinical trials. There are currently welldocumented OC markers and targets for TCR therapy, in particular cancer-testis antigens (CTA). These CTAs include melanoma-associated antigen 4 (MAGE-A4) and New York esophageal-1 (NY-ESO-1) (56-60).

CTAs are a group of proteins important for early organogenesis and as developmental proteins. Their expressions are highly regulated and are often switched off in adulthood, with the exception of the male germ cell population and in certain subset of cancers (61). Due to this, in particularly their tumor-restricted expression, and their abilities to induce strong in vivo immunogenicity, CTAs are now regarded as ideal targets for tumor specific IO approaches.

The Melanoma-associated antigen (MAGE)-A gene family is a group of CTA genes that encode for MAGE-A1, MAGE-A2, MAGE-A3, MAGE-A4, MAGE-A6, MAGE-A10, and MAGEA12 (51, 62). These were the earlier targets for TCR-T and are expressed at a frequency of about 1/10 000 . Unfortunately, there were cross-reactivities seen in TCR-T specific to MAGE-A peptides to related peptides in the brain and the heart. These cross-reactivities have resulted in severe immune related toxicities reported in TCR-T early phase clinical trials which included severe organ damages (such as the brain and heart) and even death (63).

NY-ESO-1 or New York esophageal squamous cell carcinoma 1 is another well-known CTAs where its expression is usually restricted to testicular germ cells and placenta trophoblasts. There was no or low expression seen in normal adult somatic cell, with re-expression observed in numerous cancer types including ovarian cancers (64)

As these CTAs are not entirely private but can still be found in normal somatic tissues, targeting these may result in minor to detrimental off-target toxicities. Furthermore, these listed CT antigens are not mutagenic proteins but re-expression of normal developmental proteins in cancer cells. In addition, these markers are not always exclusively upregulated in every OC, resulting in variable response rates. Table $\mathbf{1}$ listed all the Phase I and II clinical trials that are recruiting women with OC to assess the safety profiles of TCR-T.

\section{POTENTIAL FUTURE ROLE OF TCR-T THERAPY IN IMMUNOLOGICALLY COLD RARE TUMOR AND THE CONCEPT OF PERSONALIZED THERAPIES TARGETING TUMOR NEOANTIGENS}

TCR-T approach may be an attractive cell based therapy option for immunological "cold" tumor given the wider repertoire of targetable tumor neoantigens (compared to CAR-T cell based therapy). Despite having low TMB, each HGSOC and OCS will have tumor neoantigens (TNA), which can be identified by next generation sequencing of the tumors. There are a wider array of TNA targetable by TCR-T, as they are not restricted to the peptides presented on the surface of cancer cells, but also internal TNA as long as these are associated with high affinity to the patient's human leukocyte antigen (HLA) class I complexes. Thus, these will be presented to CD8+ T-cytotoxic cells. 
TABLE 1 | Summary of ongoing clinical trials for evaluating TCR therapy safety profiles in cancers including OC.

Study ID and description

\begin{tabular}{ccc}
$\begin{array}{c}\text { Study } \\
\text { phase }\end{array}$ & Intervention & Target \\
\hline । & TCR therapy & NY-ESO-1 \\
। & TCR therapy & NY-ESO-1 \\
I/lla & TCR therapy & NY-ESO-1 \\
I/lla & TCR therapy & NY-ESO-1 \\
& & \\
I & TCR therapy & MAGE-A4 \\
II & TCR therapy & Unspecified
\end{tabular}

NCT03017131 ("Genetically Modified T Cells and Decitabine in Treating Patients With Recurrent or Refractory Ovarian, Primary Peritoneal, or Fallopian Tube Cancer")

NCT03691376 ("Genetically Engineered Cells (NY-ESO-1 TCR Engineered T Cells and HSCs) After Melphalan Conditioning Regimen in Treating Patients With Recurrent or Refractory Ovarian, Fallopian Tube, or Primary Peritoneal Cancer")

NCT01567891 ("CT Antigen TCR-redirected T Cells for Ovarian Cancer")

NCT02650986 ("Gene-Modified T Cells with or Without Decitabine in Treating Patients with Advanced Malignancies Expressing

NY-ESO-1")

NCT02096614 ("Investigator Initiated Phase 1 Study of TBI-1201)

NCT03412877 ("Administration of Autologous T-cells Genetically Engineered to Express T-cell Receptors Reactive Against

Mutated Neoantigens in People with Metastatic Cancer")

TCR, T-cell receptor; NY-ESO-1, New York esophageal-1; MAGE-A4, melanoma-associated antigen 4.

The selection of the target TNA should not be affected by the heterogeneity or clonality of the tumor, although the selection of a truncal TNA is preferable, as tumor infiltrating activated T cells will exert a degree of bystander cytotoxic effect - a phenomenon that is well documented in CD8+ T cell activity in viral infections. The more crucial step to the process of developing a TNA-specific $\mathrm{T}$ cell therapy is the identification of a TNA-specific TCR. Once the TNA-specific TCR is identified, the TCR can be transduced into patient derived autologous $\mathrm{CD} 8+\mathrm{T}$ cells.

TNA selection, TCR identification and ex vivo genetic engineering are the key components for a successful personalized TCR-T in immunologically cold tumors, such as OC and OCS. At present, there are now technologies that enable the modification of the patient's own CD4+ and CD8+ cells' TCRs to express specific peptide enhanced affinity receptors (SPEARs) to increase the binding affinity of natural TCRs, thus overcoming their low affinities as a result of negative thymic selection during maturation of $\mathrm{T}$ cells in the thymus (51). Lastly, the reducing cost of Next Generation Sequencing will allow for faster, cheaper TNA discovery and may pave the way for personalized TCR-T.

\section{AUTHOR CONTRIBUTIONS}

GH, JO, and JW contributed to conception and design of the manuscript. GH and JW wrote the first draft of the manuscript.

\section{REFERENCES}

1. Ferlay J, Colombet M, Soerjomataram I, Mathers C, Parkin M, Piñeros M, et al. Estimating the global cancer incidence and mortality in 2018: GLOBOCAN sources and methods. Int J Cancer (2019) 144:1941-53. doi: 10.1002/ijc.31937

2. Matulonis UA, Sood K, Fallowfield H, Brooke S, Jalid K, Karlan B, et al. Ovarian cancer. Nat Rev Dis Primers (2016) 2:16061-1. doi: 10.1038/nrdp.2016.61

3. Helland A, Anglesio M, George J, Cowin P, Johnstone C, House C, et al. Deregulation of MYCN, LIN28B and LET7 in a molecular subtype of aggressive high-grade serous ovarian cancers. PloS One (2011) 6:e18064. doi: 10.1371/journal.pone.0018064

4. Kim HJ, Lee H, Kim M, Lee Y, Lee I, Lee K, et al. Prognostic assessment of sarcomatous histologic subtypes of ovarian carcinosarcoma. Obstet Gynecol Sci (2017) 60:350-6. doi: 10.5468/ogs.2017.60.4.350
$\mathrm{GH}, \mathrm{CS}, \mathrm{AP}, \mathrm{SD}, \mathrm{LD}$, and JO wrote sections of the manuscript. JW, SD, and LD contributed to the figure. All authors contributed to the article and approved the submitted version.

\section{FUNDING}

GH is funded by a School of Clinical Sciences, Monash University Clinician-Scientist Early Career Fellowship. JO is an $\mathrm{Al}$ and Val Rosenstrauss Fellow funded by the Rebecca Cooper Medical Foundation. Clare Scott is funded by the Stafford Fox Medical Research Foundation (CLS); Cancer Council Victoria (Sir Edward Dunlop Fellowship in Cancer Research to CLS); the Victorian Cancer Agency (Clinical Fellowships to CLS CRF1020, CRF16014.

\section{ACKNOWLEDGMENTS}

We would like to thank WEHI Stafford Fox Rare Cancer Program for the CIRCOS plots and support of tumor materials for our research project. This work was made possible through the Australian Cancer Research Foundation, the Victorian State Government Operational Infrastructure Support and Australian Government NHMRC IRIISS. All authors would like to thank all of the women and their families who participated in this research.

5. Rauh-Hain JA, Birrer M, Del Carmen MG. Carcinosarcoma of the ovary, fallopian tube, and peritoneum: Prognostic factors and treatment modalities. Gynecol Oncol (2016) 142:248-54. doi: 10.1016/j.ygyno.2016.06.003

6. Madariaga A, Rustin GJS, Buckanovich RJ, Trent JC, Oza AM. Wanna Get Away? Maintenance Treatments and Chemotherapy Holidays in Gynecologic Cancers. Am Soc Clin Oncol Educ Book (2019) 39:e152-66. doi: 10.1200/ EDBK_238755

7. Alatrash G, Jakher H, Stafford PD, Mittendorf EA. Cancer immunotherapies, their safety and toxicity. Expert Opin Drug Saf (2013) 12:631-45. doi: 10.1517/ 14740338.2013.795944

8. Zugazagoitia J, Guedes C, Ponce S, Ferrer I, Molina-Pinelo S, Paz-Ares L. Current Challenges in Cancer Treatment. Clin Ther (2016) 38:1551-66. doi: 10.1016/j.clinthera.2016.03.026

9. Oda K, Hamanishi J, Matsuo K, Hasegawa K. Genomics to immunotherapy of ovarian clear cell carcinoma: Unique opportunities for management. Gynecol Oncol (2018) 151:381-9. doi: 10.1016/j.ygyno.2018.09.001 
10. Gaynor N, Crown J, Collins DM. Immune checkpoint inhibitors: Key trials and an emerging role in breast cancer. Semin Cancer Biol (2020). doi: 10.1016/ j.semcancer.2020.06.016

11. Chae YK, Arya A, Iams W, Cruz M, Chandra S, Choi J, et al. Current landscape and future of dual anti-CTLA4 and PD-1/PD-L1 blockade immunotherapy in cancer; lessons learned from clinical trials with melanoma and non-small cell lung cancer (NSCLC). J Immunother Cancer (2018) 6:39. doi: 10.1186/s40425-018-0349-3

12. Sun L, Zhang L, Yu J, Zhang Y, Pang X, Ma C, et al. Clinical efficacy and safety of anti-PD-1/PD-L1 inhibitors for the treatment of advanced or metastatic cancer: a systematic review and meta-analysis. Sci Rep (2020) 10:2083. doi: 10.1038/s41598-020-58674-4

13. Ghisoni E, Imbimbo M, Zimmermann S, Valabrega G. Ovarian Cancer Immunotherapy: Turning up the Heat. Int J Mol Sci (2019) 20:2927. doi: 10.3390/ijms20122927

14. Disis ML, Taylor MH, Kelly K, Beck JT, Gordon M, Moore KM, et al. Efficacy and Safety of Avelumab for Patients With Recurrent or Refractory Ovarian Cancer: Phase 1b Results From the JAVELIN Solid Tumor Trial. JAMA Oncol (2019) 5:393-401. doi: 10.1001/jamaoncol.2018.6258

15. Hamanishi J, Mandai M, Ikeda T, Minami M, Kawaguchi A, Murayama T, et al. Safety and Antitumor Activity of Anti-PD-1 Antibody, Nivolumab, in Patients With Platinum-Resistant Ovarian Cancer. J Clin Oncol (2015) 33:4015-22. doi: 10.1200/JCO.2015.62.3397

16. Liu JF, Gordon M, Veneris J, Braiteh F, Balmanoukian A, Eder JP, et al. Safety, clinical activity and biomarker assessments of atezolizumab from a Phase I study in advanced/recurrent ovarian and uterine cancers. Gynecol Oncol (2019) 154:314-22. doi: 10.1016/j.ygyno.2019.05.021

17. Farkkila A, Gulhan DC, Casado J, Jacobson CA, Nguyen H, Kochupurakkal B, et al. Immunogenomic profiling determines responses to combined PARP and PD-1 inhibition in ovarian cancer. Nat Commun (2020) 11:1459. doi: 10.1038/ s41467-020-15315-8

18. Conway JR, Kofman E, Mo SS, Elmarakeby H, Van Allen E. Genomics of response to immune checkpoint therapies for cancer: implications for precision medicine. Genome Med (2018) 10:93. doi: 10.1186/s13073-018-0605-7

19. Zhao S, Bellone S, Lopez S, Thakral D, Schwab C, English DP, et al. Mutational landscape of uterine and ovarian carcinosarcomas implicates histone genes in epithelial-mesenchymal transition. Proc Natl Acad Sci U S A (2016) 113:12238-43. doi: 10.1073/pnas.1614120113

20. Bowtell DD, Bohm S, Ahmed A, Aspuria PJ, Bast RC, Beral V, et al. Rethinking ovarian cancer II: reducing mortality from high-grade serous ovarian cancer. Nat Rev Cancer (2015) 15:668-79. doi: 10.1038/nrc4019

21. Bowtell DD. The genesis and evolution of high-grade serous ovarian cancer. Nat Rev Cancer (2010) 10:803-8. doi: 10.1038/nrc2946

22. Schlacher K, Christ N, Siaud N, Egashira A, Wu H, Jasin M. Double-strand break repair-independent role for BRCA2 in blocking stalled replication fork degradation by MRE11. Cell (2011) 145:529-42. doi: 10.1016/ j.cell.2011.03.041

23. Cancer Genome Atlas Research, N. Integrated genomic analyses of ovarian carcinoma. Nature (2011) 474:609-15. doi: 10.1038/nature10166

24. Ciriello G, Miller ML, Aksoy BA, Senbabaoglu Y, Schultz N, Sander C. Emerging landscape of oncogenic signatures across human cancers. Nat Genet (2013) 45:1127-33. doi: 10.1038/ng.2762

25. Martins CP, Santiago ID, Trinh A, Xian J, Guo A, Sayal K. Combined image and genomic analysis of high-grade serous ovarian cancer reveals PTEN loss as a common driver event and prognostic classifier. Genome Biol (2014) 15:526. doi: 10.1186/s13059-014-0526-8

26. Patch AM, Christie EL, Etemadmoghadam D, Garsed DW, George J, Fereday $\mathrm{S}$, et al. Whole-genome characterization of chemoresistant ovarian cancer. Nature (2015) 521:489-94. doi: 10.1038/nature14410

27. Nakayama N, Nakayama K, Shamima Y, Ishikawa M, Katagiri A, Iida K, et al. Gene amplification CCNE1 is related to poor survival and potential therapeutic target in ovarian cancer. Cancer (2010) 116:2621-34. doi: $10.1002 /$ cncr.24987

28. Etemadmoghadam D, deFazio A, Beroukhim R, Mermel C, George J, Getz G, et al. Integrated Genome-Wide DNA Copy Number and Expression Analysis Identifies Distinct Mechanisms of Primary Chemoresistance in Ovarian Carcinomas. Clin Cancer Res (2009) 15:1417-27. doi: 10.1158/1078-0432. CCR-08-1564
29. Gotoh O, Sugiyama Y, Takazawa Y, Kato K, Tanaka N, Omatsu K, et al. Clinically relevant molecular subtypes and genomic alteration-independent differentiation in gynecologic carcinosarcoma. Nat Commun (2019) 10:4965. doi: 10.1038/s41467-019-12985-x

30. Barker HE, Scott CL. Genomics of gynaecological carcinosarcomas and future treatment options. Semin Cancer Biol (2020) 61:110-20. doi: 10.1016/ j.semcancer.2019.10.006

31. Melendez B, Van Campenhout C, Rorive S, Remmelink M, Salmon I, D'Haene N. Methods of measurement for tumor mutational burden in tumor tissue. Transl Lung Cancer Res (2018) 7:661-7. doi: 10.21037/tlcr.2018.08.02

32. Samstein RM, Lee CH, Shoushtari AN, Hellmann MD, Shen R, Janjigian YY, et al. Tumor mutational load predicts survival after immunotherapy across multiple cancer types. Nat Genet (2019) 51:202-6. doi: 10.1038/s41588-0180312-8

33. Maleki Vareki S. High and low mutational burden tumors versus immunologically hot and cold tumors and response to immune checkpoint inhibitors. J Immunother Cancer (2018) 6:157. doi: 10.1186/s40425-018-0479-7

34. Buckanovich RJ, Facciabene A, Kim S, Benencia F, Sasaroli D, Balint K, et al. Endothelin B receptor mediates the endothelial barrier to $\mathrm{T}$ cell homing to tumors and disables immune therapy. Nat Med (2008) 14:28-36. doi: 10.1038/ nm1699

35. Motz GT, Santoro SP, Wang LP, Garrabrant T, Lastra RR, Hagemann IS, et al. Tumor endothelium FasL establishes a selective immune barrier promoting tolerance in tumors. Nat Med (2014) 20:607-15. doi: 10.1038/nm.3541

36. Zhang L, Conejo-Garcia JR, Katsaros D, Gimotty PA, Massobrio M, Regnani G, et al. Intratumoral T Cells, Recurrence, and Survival in Epithelial Ovarian Cancer. N Engl J Med (2003) 348:203. doi: 10.1056/NEJMoa020177

37. Peng D, et al. Epigenetic silencing of TH1-type chemokines shapes tumour immunity and immunotherapy. Nature (2015) 527:249-53. doi: 10.1038/ nature 15520

38. Zhang AW, McPherson A, Milne K, Kroeger DR, Hamilton PT, Miranda A, et al. Interfaces of Malignant and Immunologic Clonal Dynamics in Ovarian Cancer. Cell (2018) 173:1755-69.e1722. doi: 10.1016/j.cell.2018.03.073

39. McAlpine JN, Porter H, Kobel M, Nelson BH, Prentice LM, Kalloger SE, et al. BRCA1 and BRCA2 mutations correlate with TP53 abnormalities and presence of immune cell infiltrates in ovarian high-grade serous carcinoma. Mod Pathol (2012) 25:740-50. doi: 10.1038/modpathol.2011.211

40. Ovarian Tumor Tissue Analysis (OTTA) Consortium. Dose-Response Association of CD8+ Tumor-Infiltrating Lymphocytes and Survival Time in High-Grade Serous Ovarian Cancer. JAMA Oncol (2017) 3:e173290. doi: 10.1001/jamaoncol.2017.3290

41. Perica K, Varela JC, Oelke M, Schneck J. Adoptive T cell immunotherapy for cancer. Rambam Maimonides Med J (2015) 6:e0004. doi: 10.5041/ RMMJ.10179

42. Sadelain M, Riviere I, Riddell S. Therapeutic T cell engineering. Nature (2017) 545:423-31. doi: 10.1038/nature22395

43. Robbins PF, Morgan RA, Feldman SA, Yang JC, Sherry RM, Dudley ME, et al. Tumor regression in patients with metastatic synovial cell sarcoma and melanoma using genetically engineered lymphocytes reactive with NY-ESO-1. J Clin Oncol (2011) 29:917-24. doi: 10.1200/JCO.2010.32.2537

44. Chapuis AG, Egan DN, Bar M, Schmitt TM, McAfee MS, Paulson KG, et al. T cell receptor gene therapy targeting WT1 prevents acute myeloid leukemia relapse post-transplant. Nat Med (2019) 25:1064-72. doi: 10.1038/s41591019-0472-9

45. Kalos M, Levine BL, Porter DL, Katz S, Grupp SA, Bagg A, et al. T cells with chimeric antigen receptors have potent antitumor effects and can establish memory in patients with advanced leukemia. Sci Trans Med (2011) 3:95ra73. doi: 10.1126/scitranslmed.3002842

46. Ruella M, Kenderian SS. Next-Generation Chimeric Antigen Receptor T-Cell Therapy: Going off the Shelf. BioDrugs (2017) 31:473-81. doi: 10.1007/ s40259-017-0247-0

47. Neelapu SS, Locke FL, Bartlett NL, Lekakis LJ, Miklos DB, Jacobson CA, et al. Axicabtagene Ciloleucel CAR T-Cell Therapy in Refractory Large B-Cell Lymphoma. N Engl J Med (2017) 377:2531-44. doi: 10.1056/NEJMoa1707447

48. Kato D, Yaguchi T, Iwata T, Morii K, Nakagawa T, Nishimura R, et al. Prospects for personalized combination immunotherapy for solid tumors based on adoptive cell therapies and immune checkpoint blockade therapies. Jpn J Clin Immunol (2017) 40:68-77. doi: 10.2177/jsci.40.68 
49. Zhao L, Cao YJ. Engineered T Cell Therapy for Cancer in the Clinic. Front Immunol (2019) 10:2250. doi: 10.3389/fimmu.2019.02250

50. Yan W, Hu H, Tang B. Advances Of Chimeric Antigen Receptor T Cell Therapy In Ovarian Cancer. Onco Targets Ther (2019) 12:8015-22. doi: 10.2147/OTT.S203550

51. Zhang J, Wang L. The Emerging World of TCR-T Cell Trials Against Cancer: A Systematic Review. Technol Cancer Res Treat (2019) 18:1533033819831068. doi: 10.1177/1533033819831068

52. Montesion M, Murugesan K, Jin DX, Sharaf R, Sanchez N, Guria A, et al. Somatic HLA Class I Loss Is a Widespread Mechanism of Immune Evasion Which Refines the Use of Tumor Mutational Burden as a Biomarker of Checkpoint Inhibitor Response. Cancer Discov (2021) 11:282-92. doi: 10.1158/2159-8290.CD-20-0672

53. Harris DT, Kranz DM. Adoptive T Cell Therapies: A Comparison of T Cell Receptors and Chimeric Antigen Receptors. Trends Pharmacol Sci (2016) 37:220-30. doi: 10.1016/j.tips.2015.11.004

54. Kim TS, Shin EC. The activation of bystander CD8(+) T cells and their roles in viral infection. Exp Mol Med (2019) 51:1-9. doi: 10.1038/s12276-019-0316-1

55. Klampatsa A, Leibowitz MS, Sun J, Liousia M, Arguiri E, Albelda SM, et al. Analysis and Augmentation of the Immunologic Bystander Effects of CAR T Cell Therapy in a Syngeneic Mouse Cancer Model. Mol Ther Oncolytics (2020) 18:360-71. doi: 10.1016/j.omto.2020.07.005

56. Bellati F, Napoletano C, Tarquini E, Palaia I, Landi R, Manci N, et al. Cancer testis antigen expression in primary and recurrent vulvar cancer: association with prognostic factors. Eur J Cancer (2007) 43:2621-7. doi: 10.1016/ j.ejca.2007.08.031

57. Odunsi K, Jungbluth AA, Stockert E, Qian F, Gnjatic S, Tammela J, et al. NYESO-1 and LAGE-1 cancer-testis antigens are potential targets for immunotherapy in epithelial ovarian cancer. Cancer Res (2003) 63:6076-83.

58. Chitale DA, Jungbluth AA, Marshall DS, Leitao MM, Hedvat CV, Kolb D, et al. Expression of cancer-testis antigens in endometrial carcinomas using a tissue microarray. Mod Pathol (2005) 18:119-26. doi: 10.1038/ modpathol.3800232
59. Napoletano C, Bellati F, Tarquini E, Tomao F, Taurino F, Spagnoli G, et al. MAGE-A and NY-ESO-1 expression in cervical cancer: prognostic factors and effects of chemotherapy. Am J Obstet Gynecol (2008) 198:99-e91. doi: 10.1016/ j.ajog.2007.05.019

60. Hassan R, Kreitman RJ, Pastan I, Willingham MC. Localization of mesothelin in epithelial ovarian cancer. Appl Immunohistochem Mol Morphol (2005) 13:243-7. doi: 10.1097/01.pai.00000141545.36485.d6

61. Gibbs ZA, Whitehurst AW. Emerging Contributions of Cancer/Testis Antigens to Neoplastic Behaviors. Trends Cancer (2018) 4:701-12. doi: 10.1016/j.trecan.2018.08.005

62. Serrano A, Lethé B, Delroisse J-M, Lurquin C, De Plaen E, Brasseur F, et al. Quantitative Evaluation of the Expression of Mage Genes In Tumors By Limiting Dilution of Cdna Libraries. Int J Cancer (1999) 83:664-9. doi: 10.1002/(SICI)1097-0215(19991126)83:5<664::AID-IJC16>3.0.CO;2-V

63. Morgan RA, Chinnasamy N, Abate-Daga D, Gros A, Robbins PF, Zheng Z, et al. Cancer regression and neurological toxicity following anti-MAGE-A3 TCR gene therapy. J Immunother (2013) 36:133-51. doi: 10.1097/ CJI.0b013e3182829747

64. Thomas R, Al-Khadairi G, Roelands J, Hendrickx W, Dermime S, Bedognetti D, et al. NY-ESO-1 Based Immunotherapy of Cancer: Current Perspectives. Front Immunol (2018) 9:947. doi: 10.3389/fimmu.2018.00947

Conflict of Interest: The authors declare that the research was conducted in the absence of any commercial or financial relationships that could be construed as a potential conflict of interest.

Copyright $\odot 2021 \mathrm{Wu}$, Dand, Doig, Papenfuss, Scott, Ho and Ooi. This is an openaccess article distributed under the terms of the Creative Commons Attribution License (CC BY). The use, distribution or reproduction in other forums is permitted, provided the original author(s) and the copyright owner(s) are credited and that the original publication in this journal is cited, in accordance with accepted academic practice. No use, distribution or reproduction is permitted which does not comply with these terms. 